Published in final edited form as:

Pediatr Obes. 2017 February ; 12(1): 28-37. doi:10.1111/ijpo.12102.

\title{
LA Sprouts Randomized Controlled Nutrition, Cooking and Gardening Program Reduces Obesity and Metabolic Risk in Hispanic/Latino Youth
}

\author{
Nicole M. Gatto, MPH, PhD $^{1}$, Lauren C. Martinez ${ }^{2}$, Donna Spruijt-Metz, MA, $\mathrm{PhD}^{2,3,4}$, and \\ Jaimie N. Davis, RD, PhD $^{5}$ \\ ${ }^{1}$ Center for Nutrition, Healthy Lifestyles \& Disease Prevention, School of Public Health, Loma \\ Linda University \\ ${ }^{2}$ Department of Preventive Medicine, University of Southern California \\ ${ }^{3}$ Center for Economic and Social Research, University of Southern California \\ ${ }^{4}$ Department of Psychology, University of Southern California \\ ${ }^{5}$ Department of Nutritional Sciences, University of Texas at Austin
}

\begin{abstract}
Objectives-Explore the effects of a novel 12-week gardening, nutrition, and cooking intervention ("LA Sprouts") on dietary intake, obesity parameters and metabolic disease risk among low-income, primarily Hispanic/Latino youth in Los Angeles.

Methods-Randomized control trial involving four elementary schools [2 randomized to intervention $\left(172,3^{\text {rd }}-5^{\text {th }}\right.$ grade students); 2 randomized to control $\left(147,3^{\text {rd }}-5^{\text {th }}\right.$ grade students $\left.)\right]$. Classes were taught in 90-minute sessions once/week for 12 weeks. Data collected at pre- and post-intervention included dietary intake via food frequency questionnaire (FFQ), anthropometric measures [BMI, waist circumference (WC)], body fat, and fasting blood samples.
\end{abstract}

Results-LA Sprouts participants compared to controls had significantly greater reductions in BMI z-scores ( -0.1 vs. -0.04 , respectively; $\mathrm{p}=0.01)$ and WC $(-1.2$ vs. $0.1 \mathrm{~cm} ; \mathrm{p}<0.001)$. Fewer LA Sprouts participants had the metabolic syndrome (MetSyn) after the intervention than before, while controls with MetSyn increased. LA Sprouts participants compared to controls increased dietary fiber intake $(+3.4 \%$ vs. $-16.5 \%$; $\mathrm{p}=0.04)$. All participants decreased vegetable intake, but decreases were less in LA Sprouts than controls $(-3.7 \%$ vs. $-26.1 \%$; $\mathrm{p}=0.04)$. Change in fruit intake did not differ between LA Sprouts and controls.

Corresponding Author: Nicole M. Gatto, MPH, PhD, Associate Professor of Epidemiology, Center for Nutrition, Healthy Lifestyles \& Disease Prevention, Loma Linda University | School of Public Health, 24951 North Circle Drive, Nichol Hall 2025, Loma Linda, California 92350, Office: (909) 558-7597 Cell: (323) 244-6039, ngatto@llu.edu.

CONFLICTS OF INTEREST

No author has any financial interest or conflict of interest to disclose.

AUTHOR CONTRIBUTIONS: Authors NMG and JND designed and conducted the research and have primary responsibility for final content. NMG and LCM analyzed data and performed statistical analysis. NMG, JND, LCM and DSM wrote the paper.

ClinicalTrials.gov identifier NCT02291146. 
Conclusions-LA Sprouts was effective in reducing obesity and metabolic risk, however, additional larger and longer term studies are warranted.

\section{Keywords}

Latino; Hispanic; children; childhood obesity; BMI; waist circumference; fruit and vegetable consumption; gardening

\section{INTRODUCTION}

The increased prevalence of childhood obesity in the US is concerning, and has led to projections that one in three male and two in five female children born in the year 2000 will develop diabetes in their lifetime[1]. Nearly one-third(31.8\%) of US children and adolescents aged 2-19 years were overweight or obese in 2011-2012, including $16.9 \%$ who were obese[2]. Pediatric obesity is associated with an increase in cardiovascular disease(CVD) risk factors, asthma, and psychological problems during childhood[3].

Adolescent Hispanic/Latinos have higher obesity rates than their Caucasian counterparts[2]. Low intakes of dietary fiber, specifically from fruits and vegetables, coupled with high consumption of refined grains and added sugar are linked to obesity and related disorders in Hispanic/Latino youth aged 8-18 years in Los Angeles(LA)[4], highlighting modifiable dietary behaviors to be targeted in interventions[4].

Socioeconomic status(SES) is an important determinant of access to healthy, high-quality fresh fruits, vegetables and other foods[5]; low SES residents of "food desert" neighborhoods in urban areas are less likely to have access than residents of higher SES neighborhoods.

Food preferences are shaped when children are young, and children's preferences for vegetables are strong predictors of consumption[6]. Having direct experiences with growing food enhances children's understanding of foods and their relationship to health[6]. Many programs for children that involve gardening and nutrition components exist[7-12], and many have led to improvements in fruit but not vegetable consumption[13]. However, none have included experimental designs allowing more rigorous evaluation of their impact on obesity and metabolic risk factors[14].

In 2010, a non-randomized 12-week after-school gardening, nutrition/cooking intervention called "LA Sprouts" was pilot-tested in predominantly low-income Hispanic/Latino elementary school children in LA, and was associated with reductions in body mass index(BMI) and systolic blood pressure(SBP)[7], and with increases in dietary fiber intake and preferences for vegetables[7,15]. These preliminary findings led to the development and implementation of a small randomized control trial(RCT) of LA Sprouts during 2012-2014 in this population[16]. This novel study hypothesizes that LA Sprouts participants compared to controls would experience reductions in adiposity and metabolic risk factors and increases in intake of fruit, vegetables and overall dietary fiber. 


\section{METHODS}

\section{Participants}

A description of the LA Sprouts study design and eligibility criteria is provided elsewhere[16]. Briefly, during 2011-2013, four elementary schools in Los Angeles Unified School District (LAUSD) were identified as eligible because they 1) offered an after-school program ("LA's BEST"), 2) had a student body $\geq 75 \%$ Latino, 3) had $\geq 75 \%$ of students participating in the free lunch program, 4) were within 10 miles of University of Southern California(USC), 5) were interested in a school garden/hosting a gardening program, and 6) could make an administrative commitment. Students at two schools were randomly assigned to receive the intervention and students at two served as controls. All $3^{\text {rd }}, 4^{\text {th }}$ and $5^{\text {th }}$ grade students enrolled in LA's BEST were invited to participate (Figure 1). Institutional Review Boards of USC, the University of Texas at Austin, Loma Linda University, and LAUSD approved the study. Informed written consent from parents and assent from children were obtained.

\section{Description of the Intervention}

LA Sprouts was taught in school gardens constructed on campus[16]. Raised bed planter boxes were placed on unpaved, grassy areas of the school yard or on areas where asphalt was removed; gardening tools were provided. An outdoor modular kitchen was outfitted with cooking supplies. Classes were held once a week for 12 weeks during either the fall or winter/spring school semester. Separate classes were offered to each grade level. The classes consisted of a 45-minute interactive cooking/nutrition lesson and a 45-minute gardening lesson taught by an educator with a nutrition or gardening background. The program's curriculum and theoretical framework based in part on Bandura's "self-efficacy"[17] are described elsewhere[7, 15, 16]. Students worked in small teams led by the educator to cook/ prepare a snack recipe each week that emphasized fruit and/or vegetable ingredients. Gardening activities also used a "hands-on" approach, where children participated in planting, growing and harvesting organic fruits and vegetables. Parallel classes were offered to parents bimonthly on mornings, evenings and weekends.

\section{Description of Control Group}

Students at two control schools did not receive any nutrition, cooking, or gardening information from investigators between pre- and post-testing, and schools were asked to refrain from augmenting their curriculum with similar lessons during the study period. Following post-testing, control schools received a delayed LA Sprouts intervention, including a school garden.

Data Collection occurred during the week prior to instruction (baseline measures) or 7-14 days after the final day of instruction (for post-intervention). Study personnel who were not blinded to group assignment were trained to perform data collection using standardized protocols. Staff were directed to review protocols; participated in demonstrations by the principal investigator $(\mathrm{PI})$ and or project manager (PM); and were observed to ensure proper technique. A PI or PM was present to supervise data collection. 


\section{Anthropometric and Metabolic Disease}

Height was measured with a free-standing stadiometer(Seca, Birmingham, UK) and weight and percent body fat via bioelectrical impedance(Tanita TBF 300A, Arlington Heights, IL). BMI z-scores and percentiles were determined using CDC cut-points for age and sex. Blood pressure(BP) was measured with an automated monitor with appropriate child cuffs (Omron, Schaumberg, IL); waist circumference(WC) measures followed NHANES protocol[18].

The Child Questionnaire included items on demographics and SES. Dietary intake was measured using the Block Kids Food Screener ("last week" version). This 41-item screener was developed and adapted from the Block Kids 2004 FFQ (NutritionQuest, Berkeley, CA), and is validated in metropolitan area youth[19]. NutritionQuest uses screener data to derive variables reflecting nutrient, food and food group estimates.

Optional Fasting Blood Samples were collected off-campus in the morning before school or on weekends by licensed phlebotomists with experience drawing blood in overweight children. Samples were processed and stored at USC until they were delivered to the Ritchey lab at USC (glucose and insulin) and the Gower lab at the University of Alabama (lipids) for analysis.

Glucose was assayed using a Yellow Springs Instruments analyzer (Yellow Springs, $\mathrm{OH}$ ). Total cholesterol, high-density lipoprotein cholesterol (HDL), and triglyceride levels were measured using enzymatic methods on a Stanbio Sirrus analyzer (Stanbio Laboratory, Boerne, TX); Low-density lipoprotein (LDL) was calculated using the Friedwald equation. Insulin was quantified using an ELISA kit (EMD Millipore, St. Charles, MO). Homeostatic model assessment (HOMA-IR) was calculated as a measure of insulin resistance.

Metabolic Syndrome(MetSyn) was identified using the definition of Cook et al. which was adapted in adolescents using the National Cholesterol Education Program's (NCEP) criteria for adults[20].

\section{Statistical Analyses}

Preliminary Analyses-Anthropometric and metabolic data were screened for plausibility by conducting residual analyses examining how the baseline value of a given variable predicted that at follow-up. Original data was checked to resolve possible measurement errors for participants with standardized residuals $>|3|$, otherwise if errors could not be corrected and it was concluded that the observation was an outlier, it was removed from analyses. Dietary screener variables were selected as relevant to study hypotheses. Excluded as implausible or outlying were observations for which the change in reported intake between pre- and post-intervention was $\leq$ the $1^{\text {st }}$, or $\geq$ the $99^{\text {th }}$ percentile. The number of observations set to missing varied by the dependent variable, yet fewer than $2.2 \%$ of observations were excluded. Variables were examined for normality and data transformations were attempted for SBP, HDL and fasting insulin, but improvements were not substantial. Thus, analyses used the original data.

Primary Analyses-Frequencies were tabulated for categorical socio-demographic variables at baseline; mean \pm standard errors (SE) for continuous variables at baseline and 
follow-up were calculated, adjusting for age (continuous), sex, Hispanic/Latino (yes, no), English spoken at home (yes, no), school (Monte Vista, Loreto, Sierra Park, Euclid). Means for nutrients and foods/food groups were additionally adjusted for energy intake (kcal). Repeated measures mixed effects linear models with school as a random effect and a variance components covariance structure assessed whether adjusted mean changes in anthropometric, clinical and dietary variables over the 12-week study period differed between intervention and control groups. Sensitivity analyses examined the effect of additional adjustment for baseline BMI in models where dietary variables and clinical variables were the dependent variable. Analyses were conducted in the overweight/obese subsample to examine whether results were similar to those for the total study sample. Since this study is intended to inform the development of a larger scale and longer term RCT, adjustment for multiple comparisons was not done. Because of the small size of the study (only 4 schools randomized), analyses were anticipated to be underpowered to detect differences in change measures between students in schools[21]. Calculations of intraclass correlations (ICCs) for the main outcome variables were all small in magnitude (ICCs < 0.05 ) suggesting very low degrees of clustering within schools. After verifying that demographic and anthropometric variables, plus energy intake, were not different between schools within treatment groups after randomization/prior to initiation of the intervention, unadjusted mean change scores were calculated in the outcome variables (from pre- to postintervention) for intervention and control schools. T-tests examined whether the average scores for schools were different between treatment groups. Results are provided as supplemental files (S1-S3). All analyses used SAS version 9.4 (SAS Institute Inc., Cary, NC, USA.).

\section{RESULTS}

By design[16], the study population was $\sim 89 \%$ Hispanic/Latino and $\sim 90 \%$ eligible for free lunch at school (Table 1). The majority ( $>50 \%$ ) were overweight (BMI $\geq 85$ th percentile), and more than one-third were obese (BMI $\geq 5$ th percentile), which is higher than the national average for this ethnic group[2]. Average SPB and DBP may have been higher than national averages[22], dietary fiber intake lower[23] while fruit and vegetable intake was approximately comparable[24]. LA Sprouts participants and controls did not differ at baseline in age, sex, ethnicity, BMI and most sociodemographic factors examined. There was a trend for LA Sprouts participants compared to controls to be less likely to speak English at home $(\mathrm{p}=0.06)$.

After the 12-week program, LA Sprouts participants had significantly greater reductions in BMI z-score than controls [ $-0.1(9.9 \%)$ versus $-0.04(3.8 \%)$, respectively; $\mathrm{p}=0.01]$. LA Sprouts participants had a $1.2 \mathrm{~cm}(1.7 \%)$ reduction in WC, while controls had a 0.1 $\mathrm{cm}(0.1 \%)$ increase after the intervention $(\mathrm{p}<0.001)$ (Table 2$)$. The number of students overall who fit criteria for the MetSyn was small. However, there were fewer LA Sprouts participants with the MetSyn after $(n=1)$ the intervention than before $(n=7)$, while the number of controls with the MetSyn was essentially the same between pre- $(n=3)$ and postintervention $(n=4)$. For percent body fat, SBP and DBP, and blood measures, the change between pre- and post- intervention was not statistically different between LA Sprouts participants and controls. Adjustment for BMI did not appreciably alter the change estimates 
or impact conclusions about the effect of the intervention on obesity or metabolic measures(data not shown). Results in the overweight/obese strata were similar to those in the total sample(data not shown).

LA Sprouts participants increased dietary fiber intake by $0.4 \mathrm{~g} / \mathrm{d}(3.4 \%)$, compared to a $2.0 \mathrm{~g} /$ $\mathrm{d}(16.5 \%)$ decrease in controls ( $\mathrm{p}=0.04)$ (Table 2). Both LA Sprouts participants and controls had decreased vegetable intake, but the decreases were smaller in LA Sprouts than controls [ $-0.03 \mathrm{CE} / \mathrm{d}(3.7 \%)$, versus $-0.2 \mathrm{CE} / \mathrm{d},(26.1 \%) ; \mathrm{p}=0.04]$. LA Sprouts tended to increase intake of whole grains and green beans and peas, while controls decreased their intake(p $\leq$ 0.10). Change in fruit intake overall and intake of apples, bananas and oranges did not significantly differ between LA Sprouts and control subjects.

\section{DISCUSSION}

This study suggests that the LA Sprouts program, the first randomized controlled school gardening, nutrition, and cooking intervention, was effective in reducing obesity risk in predominantly Hispanic/Latino elementary school aged children. While the reductions were relatively small in magnitude (decreases of 0.1 in BMI z-score and $1.2 \mathrm{~cm}$ in WC), it is notable that changes were observed over a 12-week period. The modest reduction in BMI may also be interpreted relative to those observed in previous more intensive RCTs of dietary modifications, rigorous nutrition education, intense and monitored physical activity sessions, or clinic-based with or without healthcare professionals, which have demonstrated inconsistent success[25]. A 12-week behavioral modification program for Hispanic/Latino children aged 7-15 years and their families that provided alternative foods to substitute for those with high glycemic index, dietary prescriptions and physical activity sessions found a 0.156 point reduction in BMI z-score after 3 months[26]. Furthermore, as the prevalence of overweight and obesity in our study population was higher than national averages[2], this suggests that even small risk reductions represent progress in tackling the problem.

LA Sprouts may have led to changes in dietary intake, with an observed increase in dietary fiber. Some[7-12], but not all[11, 27] previous non-randomized studies of school-garden based educational programs have demonstrated an effect on increasing fruit or vegetable intake in children. With over $90 \%$ of our students eligible for free or reduced cost meals, as much as $2 / 3$ of their daily dietary intake may be determined by what is offered at schools. This contextualizes an interpretation of the magnitude of change in dietary intakes associated with the intervention.

The number of students with the MetSyn was small, yet a decrease in MetSyn among LA Sprouts participants from pre- to post-intervention was observed. While this finding should be interpreted with caution, it may indicate that LA Sprouts had an effect on biochemical processes associated with this clustering of metabolic risk factors. The importance of our WC finding is further supported by the emphasis placed on the role of abdominal obesity by the International Diabetes Foundation, who advocates that WC be used to identify children aged $<10$ years to target weight reduction and be a criterion to diagnose the MetSyn for children aged $10-<16$ years. 
LA Sprouts was designed to be culturally tailored by including recipes that targeted foods familiar to the study population such as salsas and vegetable quesadillas. An effect of the intervention on fruit consumption was not observed, which is not in line with the study hypothesis but is concordant with some other non-randomized school-garden based educational interventions targeting fruit intake[9, 11,28]. The food screener did not provide a broad dietary assessment, or of fruits and vegetables which may be more commonly consumed by cultures reflected in our study population [i.e., papayas, nopales (cactus)]. Thus the null findings for fruits may reflect inadequate sensitivity of our data collection instrument. Furthermore, FFQs are not able to precisely quantify intake of nutrient consumption or differences among varieties of foods that may be captured in a single food item question. Funding, time and sample size limitations prevented collection of 24-hour dietary recalls. Nevertheless, the Block screener demonstrated good validity against 3, 24hour dietary recalls in 99 youth in a metropolitan area, with de-attenuated correlations between the two instruments ranging from 0.526 for vegetables to 0.878 for potatoes[19].

LA Sprouts was developed to take place during the after school hours because this time is ideal for implementing such health programs. Students who remain on campus after school are captive audiences for three to four hours. Data suggest that 50\% of school children in K-8th grade aged 5-13 years are regularly in non-parental care before and after school[29]. Many after-school care providers include scheduled time for enrichment in their programming and seek activities. The afterschool hours are an opportunity to enhance students' academic achievement. It is possible to incorporate fun hands-on activities such as cooking or gardening that may not be feasible in a classroom setting. Afterschool programs do not compete with required academic instruction and are not required to meet standards. Nevertheless, the LA Sprouts curriculum has been mapped on school standards (i.e., math, science, language arts, and health) and could be utilized during the school day.

Because this was a small study of 4 schools, analysis of change measures between clusters of students in schools would be underpowered[21] and the current approach may lead to false positive results. Data is not available on long-term sustainability of the program or results beyond the 12-week study period. Trained educators taught the program; it is uncertain whether similar results can be expected when taught by afterschool staff. However, several train-the-trainer workshops were held and all educational resources and supplies were provided to the schools to help sustain the program. Building partnerships with school teachers, staff, parents, and community members was a practice initiated at the outset of working with each school[16]. Because of the labor-intensiveness of the study relative to its budget, trained staff involved in collection of questionnaire and anthropometric data could not logistically be blinded to treatment assignment. However, measurement bias is not a significant concern because data collection tasks were not necessarily the same for pre and post measurements, and staff did not have knowledge of data on pre measurements when collecting post. Furthermore, the focus was on change measures, and given the above, it is difficult to imagine a scenario in which pre measures were consistently overestimated while post measures consistently underestimated in intervention subjects (i.e., for WC) with the reverse true for controls. There was a smaller sample size for optional blood measures $(<50 \%$ of the total), which led to reduced statistical power, and could explain null findings. While the importance of involving parents is recognized, parent classes were poorly 
attended. Future efforts should be directed to increasing parental support and should obtain evaluation measures on parents, as the home food environment reinforces material taught to children[30]. Gardening is a source of physical activity, and future studies may want to supplement the exercise component of their programs to include more high intensity activities such as digging and weeding, which were not emphasized in the intervention.

In conclusion, LA Sprouts, a novel school garden-based, nutrition, cooking and gardening experimental intervention resulted in a decreased risk of obesity and metabolic disease and improvements in dietary intake in high-risk Hispanic/Latino youth. These findings suggest that teaching children to grow, prepare, and eat fruits and vegetables may be an efficacious approach to reducing disease risk. These findings set the stage to evaluate whether larger and longer term gardening and nutrition interventions can lead to greater change in obesity parameters and other health effects, and to understand the sustainability of associated health benefits.

\section{Supplementary Material}

Refer to Web version on PubMed Central for supplementary material.

\section{Acknowledgments}

This study was supported by funding from the NIH (grant number 5R21DK094066). A grant from the Keck Foundation provided funding to build the school gardens.

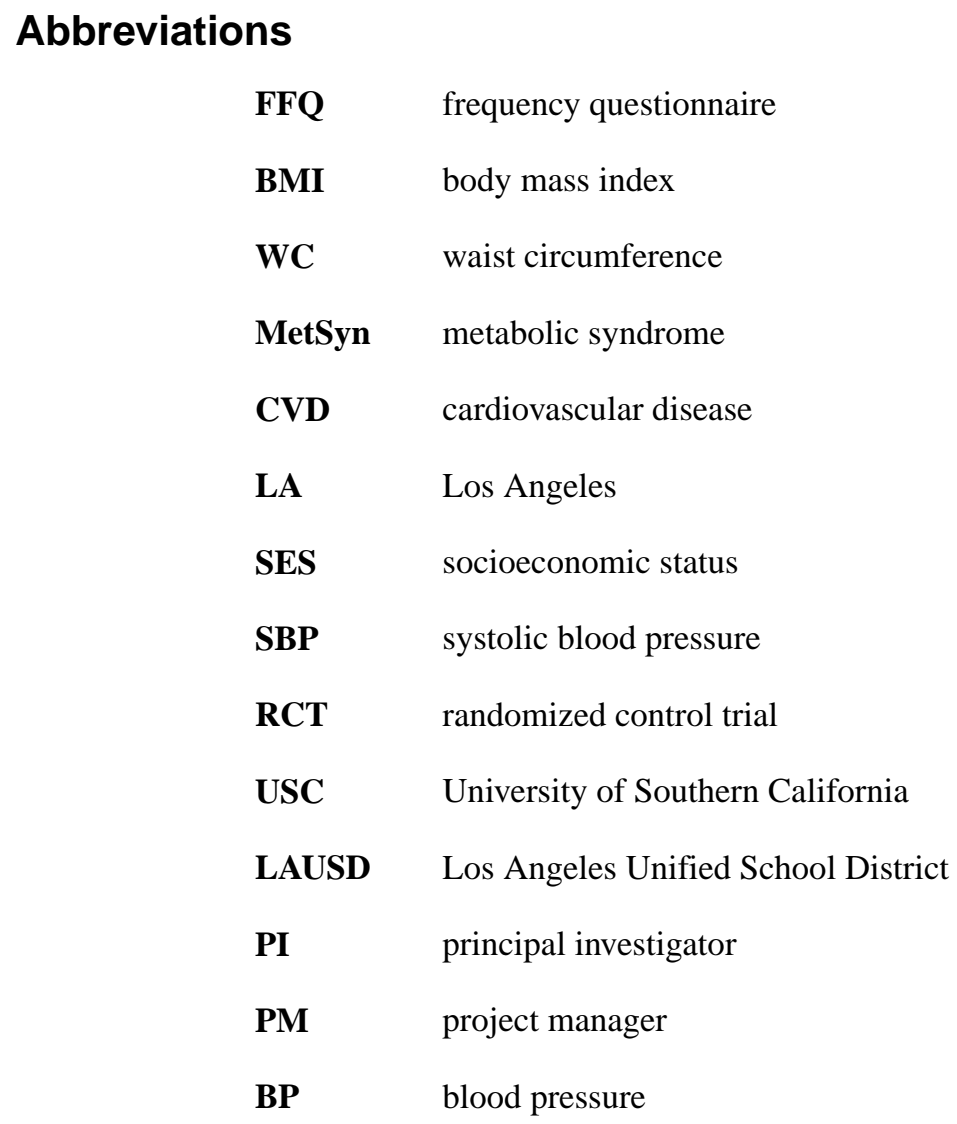

Pediatr Obes. Author manuscript; available in PMC 2018 February 01. 


\section{References}

1. Narayan KMV, Boyle JP, Thompson TJ, Sorenson SW, WDF. Lifetime risk for diabetes mellitus in the United States. Journal of American Medical Association. 2003; 290:1994-1890.

2. Ogden CL, Carroll MD, Kit BK, Flegal KM. Prevalence of childhood and adult obesity in the United States, 2011-2012. Jama. 2014; 311(8):806-14. [PubMed: 24570244]

3. Reilly JJ, Methven E, McDowell ZC, et al. Health consequences of obesity. Arch Dis Child. 2003; 88(9):748-52. [PubMed: 12937090]

4. Ventura E, Davis J, Byrd-Williams C, et al. Reduction in risk factors for type 2 diabetes mellitus in response to a low-sugar, high-fiber dietary intervention in overweight Latino adolescents. Arch Pediatr Adolesc Med. 2009; 163(4):320-7. [PubMed: 19349560]

5. Sobal, J., Stunkard, A. Influence of the home environment on the development of obesity in children. Pediatrics, 101. In: Wadden, Stunkard, editors. Handbook of obesity treatment. National Center for Health Statistics; 1998. p. 515-531.

6. Domel SB, Baranowski T, Davis HC, Thompson WO, Leonard SB, Baranowski J. A measure of stages of change in fruit and vegetable consumption among fourth- and fifth-grade school children: reliability and validity. J Am Coll Nutr. 1996; 15(1):56-64. [PubMed: 8632116]

7. Davis JN, Ventura EE, Cook LT, Gyllenhammer LE, Gatto NM. LA Sprouts: a gardening, nutrition, and cooking intervention for Latino youth improves diet and reduces obesity. J Am Diet Assoc. 2011; 111(8):1224-30. [PubMed: 21802571]

8. Heim S, Stang J, Ireland M. A garden pilot project enhances fruit and vegetable consumption among children. J Am Diet Assoc. 2009; 109(7):1220-6. [PubMed: 19559139]

9. Hermann JR, Parker SP, Brown BJ, Siewe YJ, Denney BA, Walker SJ. After-school gardening improves children's reported vegetable intake and physical activity. J Nutr Educ Behav. 2006; 38(3): 201-2. [PubMed: 16731458]

10. McAleese JD, Rankin LL. Garden-based nutrition education affects fruit and vegetable consumption in sixth-grade adolescents. J Am Diet Assoc. 2007; 107(4):662-5. [PubMed: 17383272]

11. Morgan PJ, Warren JM, Lubans DR, Saunders KL, Quick GI, Collins CE. The impact of nutrition education with and without a school garden on knowledge, vegetable intake and preferences and quality of school life among primary-school students. Public Health Nutr. 2010; 13(11):1931-40. [PubMed: 20441683]

12. Wang M, Rauzon S, Studer N, et al. Exposure to a comprehensive school intervention increases vegetable consumption. J Adolesc Health. 2010; 46:1-9. [PubMed: 20123250]

13. Evans CE, Christian MS, Cleghorn CL, Greenwood DC, Cade JE. Systematic review and metaanalysis of school-based interventions to improve daily fruit and vegetable intake in children aged 5 to 12 y. Am J Clin Nutr. 2012; 96(4):889-901. [PubMed: 22952187]

14. Davis JN, Spaniol MR, Somerset S. Sustenance and sustainability: maximizing the impact of school gardens on health outcomes. Public Health Nutr. 2015; 18(13):2358-67. [PubMed: 25704784]

15. Gatto NM, Ventura EE, Cook LT, Gyllenhammer LE, Davis JN. LA Sprouts: a garden-based nutrition intervention pilot program influences motivation and preferences for fruits and vegetables in Latino youth. J Acad Nutr Diet. 2012; 112(6):913-20. [PubMed: 22516551]

16. Martinez LC, Gatto NM, Spruijt-Metz D, Davis JN. Design and methodology of the LA Sprouts nutrition, cooking and gardening program for Latino youth: a randomized controlled intervention. Contemporary Clinical Trials. 2015; 42:219-227. [PubMed: 25896115]

17. Bandura A. Self-efficacy: toward a unifying theory of behavioral change. Psychological review. 1977; 84(2):191. [PubMed: 847061]

18. Control, C.f.D. National Health and Nutrition Examination Survey (NHANES): Anthropometry Procedures Manual. Centers for Disease Control; 2007.

19. Hunsberger M, O’Malley J, Block T, Norris JC. Relative validation of Block Kids Food Screener for dietary assessment in children and adolescents. Matern Child Nutr. 2012 
20. Cook S, Weitzman M, Auinger P, Nguyen M, Dietz WH. Prevalence of a metabolic syndrome phenotype in adolescents: findings from the third National Health and Nutrition Examination Survey, 1988-1994. Arch Pediatr Adolesc Med. 2003; 157(8):821-7. [PubMed: 12912790]

21. Murray DM, Pals SL, Blitstein JL, Alfano CM, Lehman J. Design and analysis of grouprandomized trials in cancer: a review of current practices. J Natl Cancer Inst. 2008; 100(7):483-91. [PubMed: 18364501]

22. National High Blood Pressure Education Program Working Group on High Blood Pressure in Children and Adolescents. The fourth report on the diagnosis, evaluation, and treatment of high blood pressure in children and adolescents. Pediatrics. 2004; 114(2 Suppl 4th Report):555-76. [PubMed: 15286277]

23. Storey M, Anderson P. Income and race/ethnicity influence dietary fiber intake and vegetable consumption. Nutr Res. 2014; 34(10):844-50. [PubMed: 25262170]

24. Kim SA, Moore LV, Galuska D, et al. Vital signs: fruit and vegetable intake among children United States, 2003-2010. MMWR Morb Mortal Wkly Rep. 2014; 63(31):671-6. [PubMed: 25102415]

25. Waters E, de Silva-Sanigorski A, Hall BJ, et al. Interventions for preventing obesity in children. Cochrane Database Syst Rev. 2011; (12):Cd001871. [PubMed: 22161367]

26. Mirza NM, Palmer MG, Sinclair KB, et al. Effects of a low glycemic load or a low-fat dietary intervention on body weight in obese Hispanic American children and adolescents: a randomized controlled trial. Am J Clin Nutr. 2013; 97(2):276-85. [PubMed: 23255569]

27. Christian MS, Evans CE, Nykjaer C, Hancock N, Cade JE. Evaluation of the impact of a school gardening intervention on children's fruit and vegetable intake: a randomised controlled trial. Int J Behav Nutr Phys Act. 2014; 11:99. [PubMed: 25128211]

28. Gibbs L, Staiger PK, Johnson B, et al. Expanding children's food experiences: the impact of a school-based kitchen garden program. J Nutr Educ Behav. 2013; 45(2):137-46. [PubMed: 23472931]

29. U.S. Department of Education National Center for Education Statistics. America's Children: Key National Indicators of Well-Being National Household Education Survey, as cited in Federal Interagency Forum on Child and Family Statistics. U.S. Government Printing Office; Washington, DC: 2002.

30. de Jong E, Visscher TL, HiraSing RA, Seidell JC, Renders CM. Home environmental determinants of children's fruit and vegetable consumption across different SES backgrounds. Pediatr Obes. 2015; 10(2):134-40. [PubMed: 24903612] 


\section{WHAT IS ALREADY KNOWN ABOUT THIS SUBJECT}

- $\quad$ Food preferences are shaped when children are young

- Having a direct experience with growing food enhances children's understanding of foods

- Existing programs for children that involve gardening and nutrition components have been effective at shaping attitudes and preferences

\section{WHAT THIS STUDY ADDS}

- The first randomized controlled trial of a gardening and nutrition education program on measured obesity risk

- A specific focus on a high-risk Hispanic/Latino youth population

- $\quad$ LA Sprouts was effective in reducing obesity measured by BMI and waist circumference 


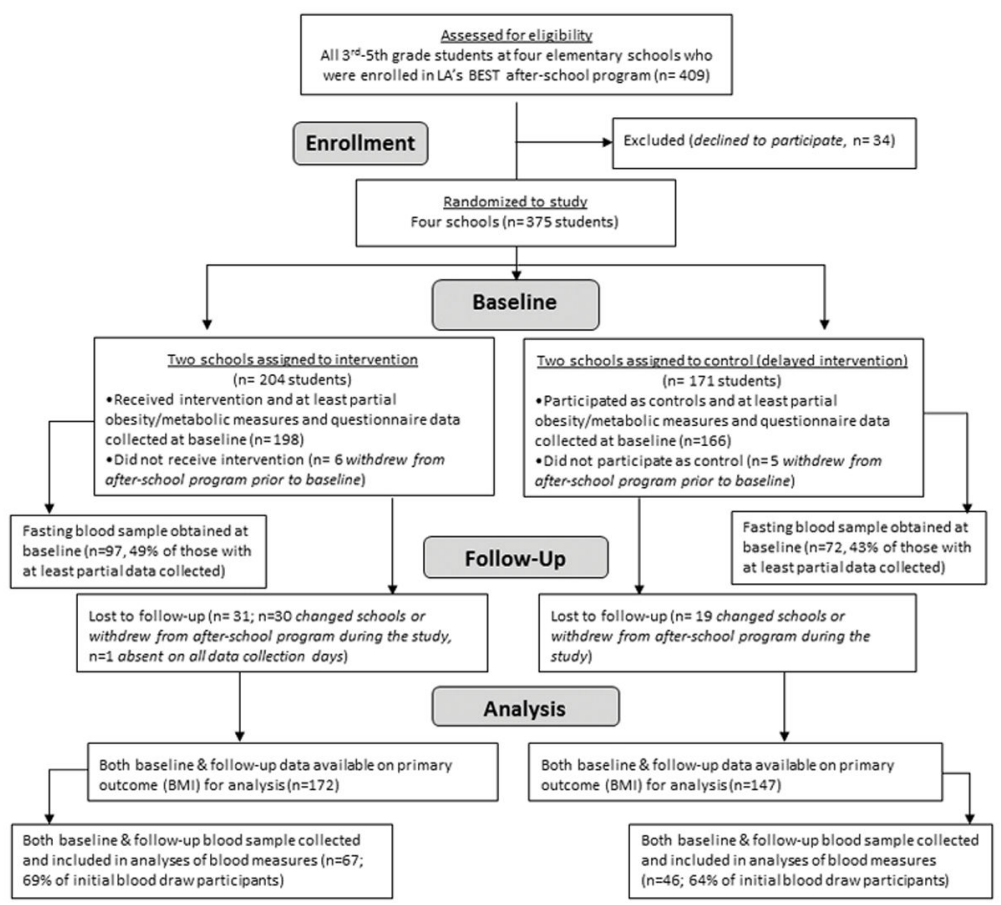

Figure 1.

Flow of participants through the LA Sprouts study 


\section{Table 1}

Demographic characteristics of LA Sprouts and control participants at baseline

\begin{tabular}{|c|c|c|c|}
\hline \multirow{2}{*}{ Characteristic, $n(\%)$ or mean \pm SD } & LA Sprouts $(n=172)$ & Controls $(n=147)$ & \multirow{2}{*}{ p-value ${ }^{a}$} \\
\hline & Pre & Pre & \\
\hline Male & $82(47.7)$ & $71(48.3)$ & 0.91 \\
\hline Hispanic/Latino $b$ & $153(89.0)$ & $127(88.8)$ & 0.97 \\
\hline Age, years & $9.3 \pm 0.9$ & $9.3 \pm 0.9$ & 0.9 \\
\hline Height, $\mathrm{cm}^{c}$ & $135.0 \pm 8.5$ & $135.0 \pm 8.5$ & 0.96 \\
\hline Weight, $\mathrm{kg}^{d}$ & $36.9 \pm 10.6$ & $38.1 \pm 12.6$ & 0.30 \\
\hline BMI, $\mathrm{kg} / \mathrm{m}^{2 i}$ & $19.8 \pm 4.1$ & $20.6 \pm 4.6$ & 0.13 \\
\hline Overweight ( 285 th percentile) $e$ & $82(51.3)$ & $73(53.3)$ & 0.73 \\
\hline Obese ( 295 th percentile) ${ }^{e}$ & $54(33.8)$ & $54(39.4)$ & 0.31 \\
\hline Systolic blood pressure, $\mathrm{mmHg} j$ & $109.4 \pm 12.1$ & $112.3 \pm 14.3$ & 0.07 \\
\hline Diastolic blood pressure, $\mathrm{mmHg} g$ & $64.3 \pm 11.1$ & $66.7 \pm 13.6$ & 0.11 \\
\hline \multicolumn{4}{|l|}{ Socioeconomic factors } \\
\hline No English spoken at home ${ }^{d}$ & $48(28.7)$ & $27(19.6)$ & 0.06 \\
\hline No computer at home ${ }^{f}$ & $42(26.1)$ & $32(23.2)$ & 0.56 \\
\hline No internet at home $g$ & $39(23.2)$ & $32(23.2)$ & 0.99 \\
\hline Mother does not have own car $g$ & $57(34.3)$ & $38(27.1)$ & 0.17 \\
\hline Eligible for free lunch at school ${ }^{h}$ & $152(90.5)$ & $125(89.3)$ & 0.73 \\
\hline
\end{tabular}

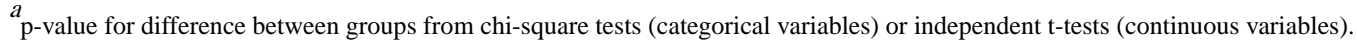

$b_{\mathrm{n}=315}$

$c_{\mathrm{n}=304}$

$d_{\mathrm{n}=305}$

$e_{\mathrm{n}=297}$

$f_{\mathrm{n}=299}$

$g_{\mathrm{n}=306}$

$h_{\mathrm{n}=308}$

$i_{\mathrm{n}=301}$

$j_{\mathrm{n}=307}$ 


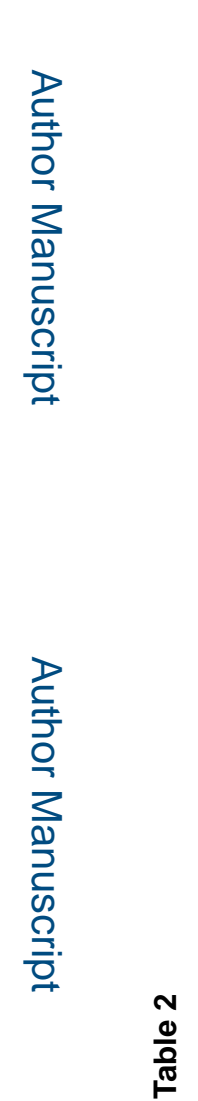

สี

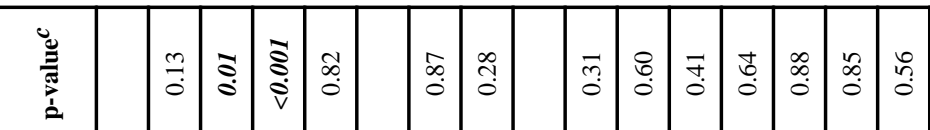

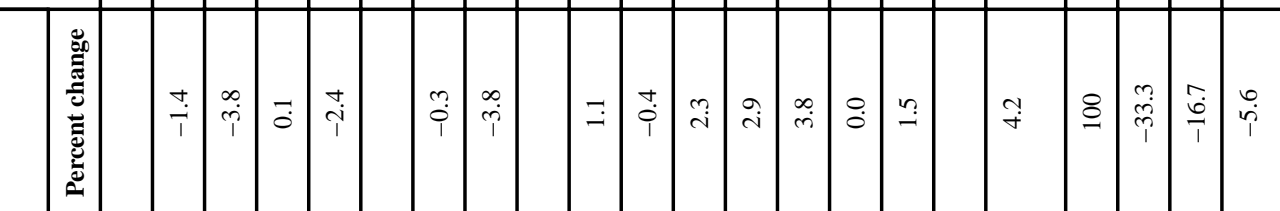

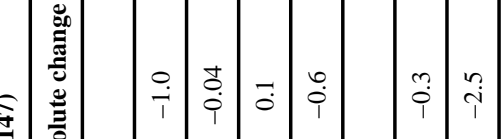

守 冚

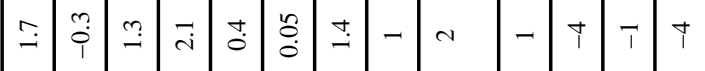

己ें

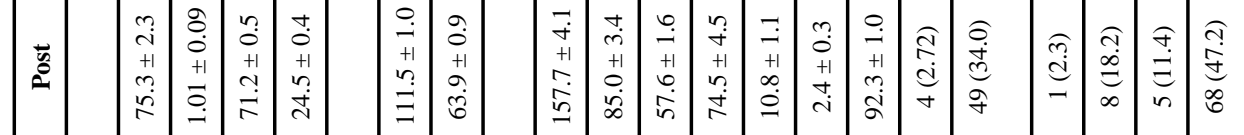

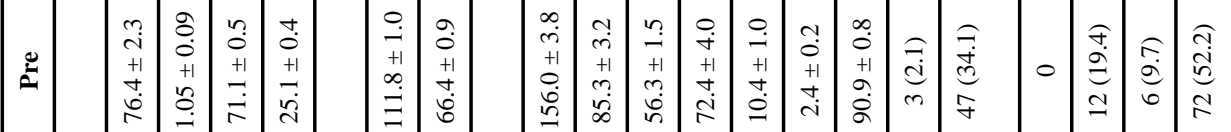

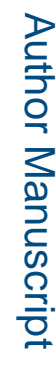

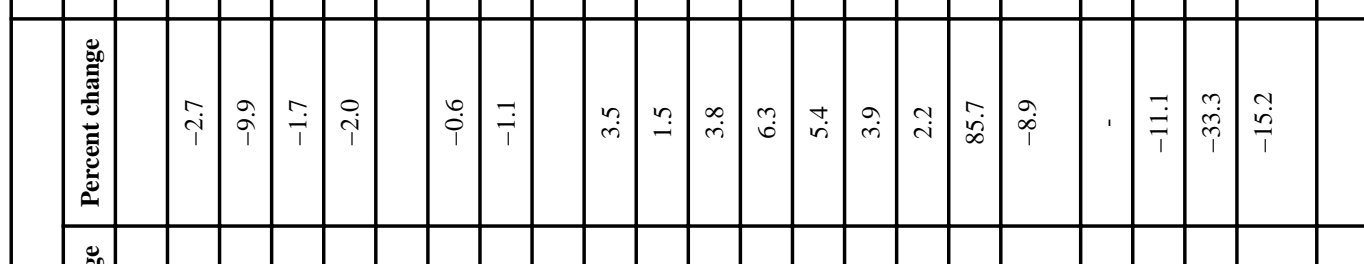

ลิ

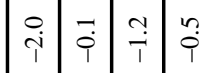

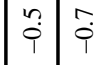

மூ)

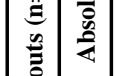

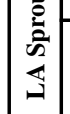

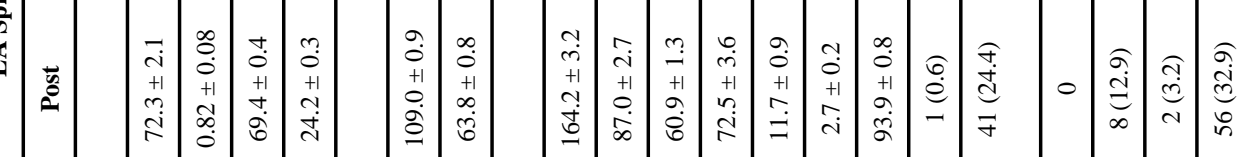

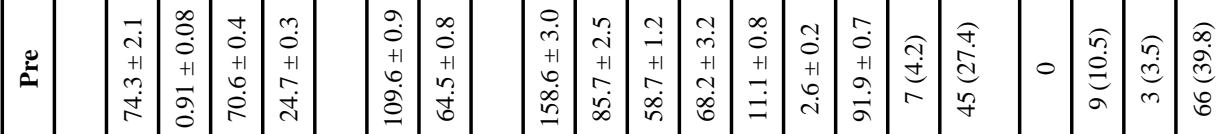

.

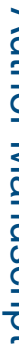

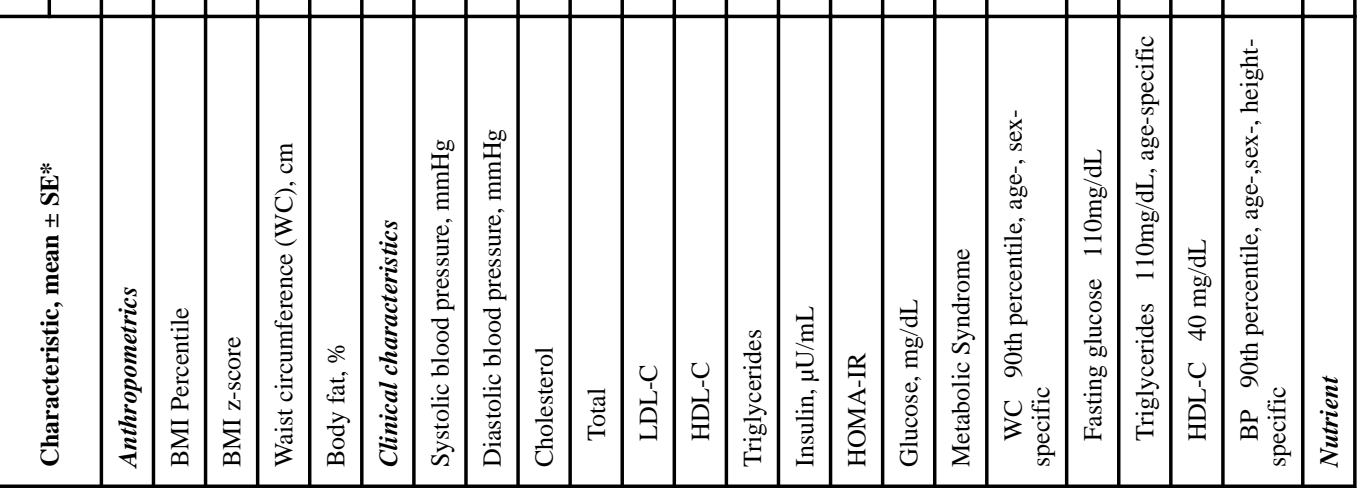

Pediatr Obes. Author manuscript; available in PMC 2018 February 01. 


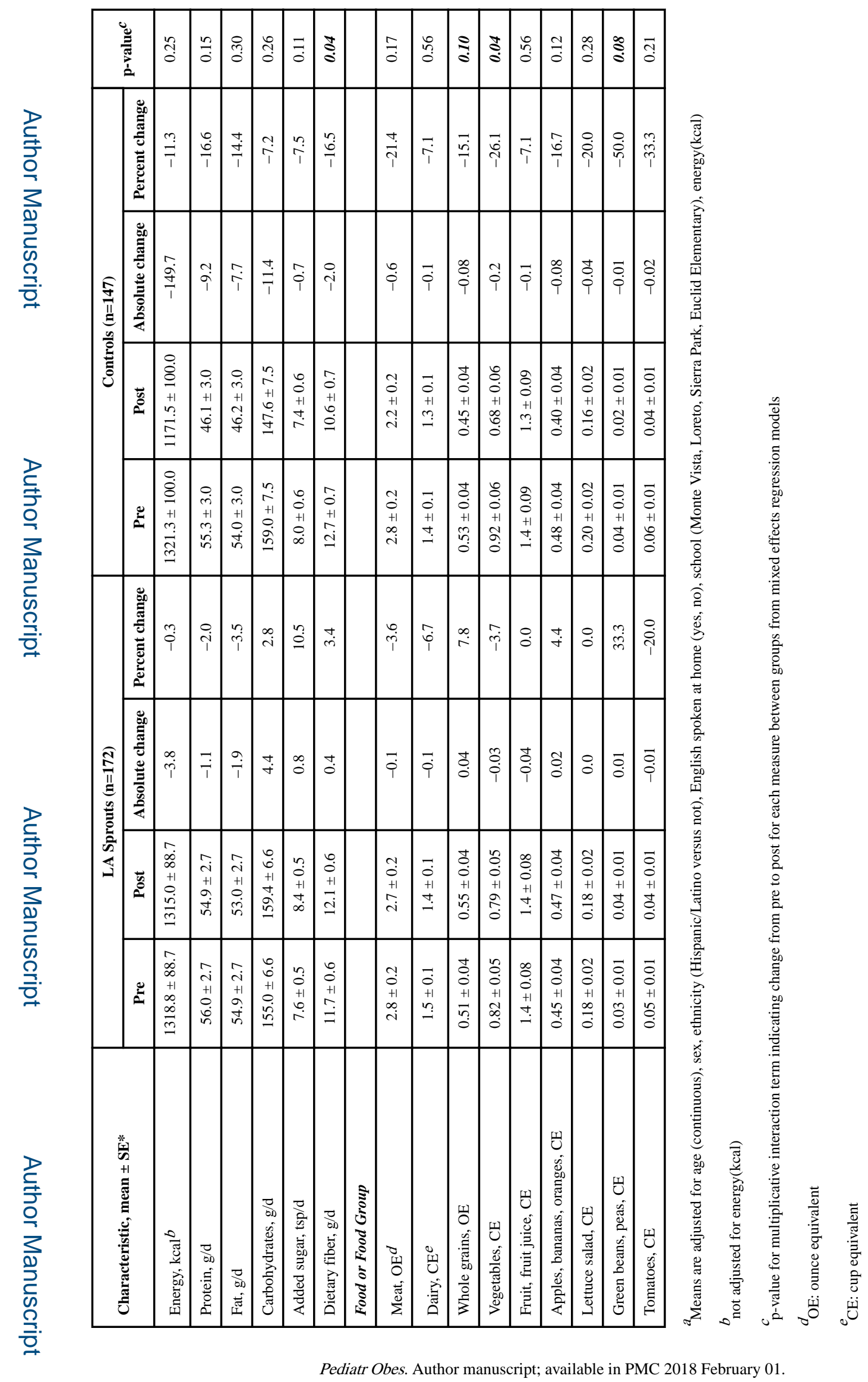

